C. Huneke and A. Vraciu

Nagoya Math. J.

Vol. 170 (2003), 175-183

\title{
SPECIAL TIGHT CLOSURE
}

\section{CRAIG HUNEKE AND ADELA VRACIU}

\begin{abstract}
We study the notion of special tight closure of an ideal and show that it can be used as a tool for tight closure computations.
\end{abstract}

\section{$\S 1$. Introduction}

Since the inception of tight closure theory, the problem of how to compute and analyze the tight closure of an ideal has been of paramount importance. The prevailing sense of how the tight closure of ideal $I$ compares to $I$ is that the extra elements needed to obtain the tight closure of $I$ are much 'deeper' in the ring than the generators of $I$. The first result of this type is due to K.E. Smith [Sm]: she proved that if $R$ is a normal graded finitely generated algebra over a perfect field of positive characteristic, $I$ is a homogeneous ideal generated by forms of degrees at least $\delta$ then every $x$ in the tight closure of $I$ but not in $I$ must have degree at least $\delta+1$. The second author of this paper was able to extend this result to nonhomogeneous ideals (still in a graded normal ring as above). This was done via a canonical decomposition of the tight closure in terms of the ideal plus another piece called the special tight closure. The purpose of this paper is to prove that the tight closure of an arbitrary ideal in a normal ring of positive characteristic with perfect residue field can be computed as the sum between the ideal and its special tight closure. Thus, the special tight closure can be envisioned as a technique for computing tight closure, or rather for imposing strong restrictions on the set of elements that can be in the tight closure. As an application, we prove that if $(R, \mathfrak{m})$ is an excellent normal local ring with perfect residue field and $\operatorname{gr}_{\mathfrak{m}} R$ is reduced, then for every ideal $I \subseteq \mathfrak{m}^{k}, I^{*} \subseteq I+\mathfrak{m}^{k+1}$, a direct generalization of Smith's result to the local case. A consequence is that every ideal that lies between $\mathfrak{m}^{k}$ and $\mathfrak{m}^{k+1}$ is tightly closed.

Received March 21, 2001.

1991 Mathematics Subject Classification: 13A35.

The authors were partially supported by the National Science Foundation. 
One would like even more precise results. For example a special case of a theorem due independently to N. Hara $[\mathrm{H}]$ and Mehta and Srinivas [MS] states that for large characteristic, if $R$ is a Cohen-Macaulay graded ring with an isolated singularity and $x_{1}, \ldots, x_{n}$ are a homogeneous system of parameters of $R$ of degrees $d_{1}, \ldots, d_{n}$, then

$$
\left(x_{1}, \ldots, x_{n}\right)^{*}=\left(x_{1}, \ldots, x_{n}\right)+R_{\geq D}
$$

where $D=d_{1}+\ldots+d_{n}$ and where $R_{\geq D}$ is the ideal generated by all forms of degree at least $D$. (See [HS] for some background information concerning this theorem.) What the best possible theorem might be remains a mystery. We begin by introducing the relevant definitions and giving some background.

Definition 1.1. Let $(R, \mathfrak{m})$ be a local Noetherian ring of characteristic $p, p$ prime, and let $I$ be an ideal. We say that an element $x \in R$ is in the special tight closure of $I$ if there exists $c \in R^{0}$ and a fixed power of $p, q_{0}$, such that $c x^{q} \in \mathfrak{m}^{\left[q / q_{0}\right]} I^{[q]}$ for all $q \geq q_{0}$, or equivalently such that $x^{q_{0}} \in\left(\mathfrak{m} I^{\left[q_{0}\right]}\right)^{*}$.

In studying tight closure, it is natural to restrict our attention to a certain class of ideals, namely those ideals that are minimal among ideals having the same tight closure.

Definition 1.2. Let $(R, \mathfrak{m})$ be local characteristic $p$ ring. An ideal $I$ of $R$ is $*$-independent if it can be generated by elements $f_{1}, \ldots, f_{n}$ (equivalently, for every minimal system of generators $\left.f_{1}, \ldots, f_{n}\right)$ such that for all $i=1, \ldots, n$ we have $f_{i} \notin\left(f_{1}, \ldots, f_{i-1}, f_{i+1}, \ldots, f_{n}\right)^{*}$.

We note the following properties of special tight closure:

Proposition 1.3. For a local ring $(R, \mathfrak{m})$ and an arbitrary ideal $I$, the following hold:

(1) $\mathfrak{m} I \subset I^{* s p} \subset I^{*}$.

(2) If $I$ is $*$-independent, $I^{* s p} \cap I=\mathfrak{m} I$.

Proof. The first inclusion in (1) follows by choosing $q_{0}=1$ in Definition 1.1, while the second inclusion follows from the definitions as well, since $c x^{q} \in m^{\left[q / q_{0}\right]} I^{[q]} \Rightarrow c x^{q} \in I^{[q]} \Rightarrow x \in I^{*}$. Part (2) is contained in Proposition 4.2 in $[\mathrm{Vr}]$. 
It is of considerable interest to identify situations in which $I^{*}$ can be recovered from $I^{* s p}$. In Theorem 4.4 in $[\mathrm{Vr}]$, it was proved that this can be accomplished if $R$ is the localization of a normal $\mathbb{N}$-graded ring at the maximal homogeneous ideal.

We now show that the assumption on the grading is unnecessary, thus the special tight closure technique for computing tight closure is available in a much larger class of rings.

The following result will be very useful. For a proof, see Proposition 2.4 in $[\mathrm{Ab}]$.

Proposition 1.4. Let $(R, \mathfrak{m})$ be a excellent, analytically irreducible local ring of characteristic $p$, let $I$ be an ideal, and let $f \in R$. Assume that $f \notin I^{*}$; then there exists $q_{0}=p^{e_{0}}$ such that for all $q \geq q_{0}$ we have $I^{[q]}: f^{q} \subset \mathfrak{m}^{\left[q / q_{0}\right]}$.

\section{$\S 2$. Main result}

THEOREM 2.1. Let $(R, \mathfrak{m})$ be a characteristic $p$ local excellent normal ring, with perfect residue field. Then $I^{*}=I+I^{* s p}$ for every ideal $I$. In particular if $I$ is *-independent, we have a direct sum decomposition

$$
\frac{I^{*}}{\mathfrak{m} I}=\frac{I}{\mathfrak{m} I} \oplus \frac{I^{* s p}}{\mathfrak{m} I} .
$$

Note that the second statement of the conclusion follows immediately from the first statement and the second part of 1.3. Before beginning the proof we need several lemmas.

LEMMA 2.2. Let $I=\left(f_{1}, \ldots, f_{n}\right)$, and $f \in I^{*}$. Assume that for all $i=1, \ldots, n$ there exists an element $\alpha_{i} \in R$ such that

$$
f_{i} \notin\left(f+\alpha_{i} f_{i}, f_{1}, \ldots, f_{i-1}, f_{i+1}, \ldots, f_{n}\right)^{*} .
$$

Then $f \in I+I^{* s p}$.

Proof. Let $f^{\prime}=f+\alpha_{1} f_{1}+\cdots+\alpha_{n} f_{n}$; clearly $f^{\prime} \in I^{*}$. We claim that for all $i=1, \ldots, n$, we have

$$
f_{i} \notin\left(f^{\prime}, f_{1}, \ldots, f_{i-1}, f_{i+1}, \ldots, f_{n}\right)^{*} .
$$

Since

$$
\left(f^{\prime}, f_{1}, \ldots, f_{i-1}, f_{i+1}, \ldots, f_{n}\right)=\left(f+\alpha_{i} f_{i}, f_{1}, \ldots, f_{i-1}, f_{i+1}, \ldots, f_{n}\right),
$$


this follows from the hypothesis.

Let $c \in R^{0}$ be such that $c\left(f^{\prime}\right)^{q} \in I^{[q]}$ for all $q$, and write

$$
c\left(f^{\prime}\right)^{q}=a_{1} f_{1}^{q}+\cdots+a_{n} f_{n}^{q} .
$$

Then there exists fixed $q_{0}=p^{e_{0}}$ such that for all $i$, we have

$$
a_{i} \in\left(\left(f^{\prime}\right)^{q}, f_{1}^{q}, \ldots, f_{i-1}^{q}, f_{i+1}^{q}, \ldots, f_{n}^{q}\right): f_{i}^{q} \subset \mathfrak{m}^{\left[q / q_{0}\right]},
$$

where the last containment follows since we have shown that $f_{i} \notin\left(f^{\prime}\right.$, $\left.f_{1}, \ldots, f_{i-1}, f_{i+1}, \ldots, f_{n}\right)^{*}$ and can apply Proposition 1.4.

The next lemma is a crucial step needed in the proof of our main theorem.

LEMMA 2.3. Let $(R, \mathfrak{m})$ be excellent normal local ring of positive characteristic $p$, and let $I=\left(f_{1}, \ldots, f_{n}\right)$ be an arbitrary ideal. If $f \in I^{*}$, there exists a test element $c$ and a power $q_{0}$ of the characteristic such that

$$
c f^{q} \in c I^{[q]}+\mathfrak{m}^{q / q_{0}} I^{[q]}
$$

for all $q \geq q_{0}$.

Proof. There is no loss of generality in assuming that $f_{1}, \ldots, f_{n}$ are $*$-independent, because otherwise we can replace them by a $*$-independent subset, generating the same ideal up to tight closure.

Since the ring is normal, the ideal defining its non-regular locus has height at least two, and moreover one can choose two elements $c, d$ in this ideal, forming a regular sequence. Theorem 6.2 in $[\mathrm{HH}]$ shows that we can replace $c$ and $d$ by some powers $c^{n}, d^{m}$ and obtain a regular sequence consisting of test elements.

Write

$$
c f^{q}=a_{1} f_{1}^{q}+\ldots+a_{n} f_{n}^{q}, \quad \text { and } \quad d f^{q}=b_{1} f_{1}^{q}+\ldots+b_{n} f_{n}^{q},
$$

with $a_{1}, \ldots, a_{n}, b_{1}, \ldots, b_{n} \in R$. Multiply the first equation by $d$ and the second one by $c$, then subtract the second equation from the first; we get:

$$
\sum_{i=1}^{n}\left(d a_{i}-c b_{i}\right) f_{i}^{q}=0
$$


Since $I$ is $*$-independent, this implies that there exists a $q_{1}$ (independent of $q$ ) such that $d a_{i}-c b_{i} \in \mathfrak{m}^{q / q_{1}} \cap(c, d)$. By the Artin-Rees lemma it follows that there exists a $q_{2} \geq q_{1}$, also independent of $q$, such that $d a_{i}-$ $c b_{i} \in \mathfrak{m}^{q / q_{2}}(c, d)$, and therefore we can write $d a_{i}-c b_{i}=c u_{i}+d v_{i}$, with $u_{i}, v_{i} \in \mathfrak{m}^{q / q_{2}}$. Because $c, d$ is a regular sequence on $R$, this implies that we can write $a_{i}=v_{i}+c g_{i}, \quad b_{i}=-u_{i}+d g_{i}$. Recalling that $c f^{q}=\sum a_{i} f_{i}^{q}$, we see that $c\left(f^{q}-\sum u_{i} f_{i}^{q}\right) \in \mathfrak{m}^{q / q_{0}} I^{[q]}$, which shows that $c f^{q} \in c I^{[q]}+\mathfrak{m}^{q / q_{0}} I^{[q]}$.

We now begin the proof of the theorem.

Proof. Let $I=\left(f_{1}, \ldots, f_{n}\right)$ and $f \in I^{*}$. There is no loss of generality in assuming that $I$ is $*$-independent. We show that one can find $\alpha_{1}, \ldots, \alpha_{n} \in$ $R$ such that the condition in Lemma 2.2 is satisfied. There is no loss of generality in working with $i=1$. Assume by contradiction that for every choice of $\alpha \in R$ we have $f_{1} \in\left(f+\alpha f_{1}, f_{2}, \ldots, f_{n}\right)^{*}$. Let $J_{\alpha}=(f+$ $\left.\alpha f_{1}, f_{2}, \ldots, f_{n}\right)$ and let $I_{0}=\left(f_{2}, \ldots, f_{n}\right)$.

Use Lemma 2.3 to write

$$
c f^{q} \equiv c u_{q} f_{1}^{q} \bmod \left(I_{0}^{[q]}, \mathfrak{m}^{q / q_{0}} f_{1}^{q}\right)
$$

for some $u_{q} \in R$.

We claim that $u_{p q} \equiv\left(u_{q}\right)^{p}(\bmod \mathfrak{m})$ for all $q \gg 0$. To prove the claim, raise Equation 1 to the $p$ th power to obtain

$$
c^{p} f^{p q} \equiv c^{p}\left(u_{q}\right)^{p} f_{1}^{p q} \bmod \left(I_{0}^{[p q]}, \mathfrak{m}^{p q / q_{0}} f_{1}^{p q}\right) .
$$

Using Equation 1 in which $q$ is replaced by $p q$, and then multiplying by $c^{p-1}$, we get

$$
c^{p} f^{p q} \equiv c^{p} u_{p q} f_{1}^{p q} \bmod \left(I_{0}^{[p q]}, \mathfrak{m}^{p q / q_{0}} f_{1}^{p q}\right) .
$$

Comparing Equations 2 and 3, we get

$$
\left[c^{p}\left(u_{p q}-\left(u_{q}\right)^{p}\right)-M\right] f_{1}^{p q} \in I_{0}^{p q},
$$

for all $q \gg 0$, where $M \in \mathfrak{m}^{p q / q_{0}}$. We obtain that

$$
c^{p}\left(u_{p q}-\left(u_{q}\right)^{p}\right)-M \in I_{0}^{p q}: f_{1}^{p q} \subseteq \mathfrak{m}^{p q / q_{1}}
$$

for some fixed $q_{1}$ and all large $q$ since $f_{1} \notin I_{0}^{*}$ (using 1.4). Then there exists a fixed constant $k$ such that

$$
c^{p}\left(u_{p q}-\left(u_{q}\right)^{p}\right) \in \mathfrak{m}^{p q / k}
$$


for all large $q$. Hence $u_{p q}-\left(u_{q}\right)^{p} \in \mathfrak{m}^{p q / k}: c^{p}$ and the latter ideal is proper for large enough $q$ since $c^{p}$ has finite order (it cannot be 0 ). Hence $u_{p q}-\left(u_{q}\right)^{p} \in \mathfrak{m}$ for all $q \gg 0$.

Since the residue field $R / \mathfrak{m}$ is perfect, we can choose $\alpha \in R$ such that $\alpha^{q} \equiv-u_{q}(\bmod \mathfrak{m})$ for all $q \gg 0$.

Using the assumption that $f_{1} \in J_{\alpha}^{*}$ and Lemma 2.3, we get

$$
c f_{1}^{q} \in\left(I_{0}^{[q]}, c\left(f^{q}+\alpha^{q} f_{1}^{q}\right), \mathfrak{m}^{q / q_{0}} f^{q}, \mathfrak{m}^{q / q_{0}} f_{1}^{q}\right) .
$$

Multiply by $c$ and use Equation 1 to obtain

$$
c^{2} f_{1}^{q} \in\left(I_{0}^{[q]}, c^{2}\left(u_{q}+\alpha^{q}\right) f_{1}^{q}, \mathfrak{m}^{q / q_{0}} f_{1}^{q}\right),
$$

and therefore we have

$$
f_{1}^{q}\left[c^{2}\left(1-B\left(u_{q}+\alpha^{q}\right)\right)-C\right] \in I_{0}^{[q]},
$$

for some $B \in R$ and $C \in \mathfrak{m}^{q / q_{0}}$.

Note that the element $c^{2}\left(1-B\left(u_{q}+\alpha^{q}\right)\right)-C$ (which multiplies $f_{1}^{q}$ into $\left.I_{0}^{[q]}\right)$ has bounded order (by the same reasoning as in the above paragraph) as $q \gg 0$ increases, because $u_{q}+\alpha^{q} \in \mathfrak{m}$ by the choice of $\alpha$. This contradicts the assumption that $I$ is $*$-independent.

\section{§3. Applications}

As an application, we obtain a generalization of one of the main results in [Sm], showing that there is an explicit lower bound (depending on the ideal) on the order of any element in the tight closure of an ideal, provided that $(R, \mathfrak{m})$ is complete normal and $\operatorname{gr}_{\mathfrak{m}} R$ is reduced.

We wish to thank the referee for suggesting the present form of the results in this section.

TheOREM 3.1. Let $(R, \mathfrak{m})$ be an excellent normal domain, with perfect residue field. Assume that there exists a filtration $\mathcal{F}=\left\{F_{k}\right\}$ consisting of $\mathfrak{m}$-primary ideals, such that the graded ring gr $\mathcal{F}=\oplus F_{k} / F_{k+1}$ is reduced. If $I$ is any ideal such that $I \subset F_{k}$, then $I^{*} \subset I+F_{k+1}$.

In particular every ideal $I$ with the property that $F_{k+1} \subset I \subset F_{k}$ for some integer value of $k$ is tightly closed.

Proof. Note that the assumption that gr $\mathcal{F}$ is reduced implies that each of the ideals $F_{k}$ is integrally closed. If $u \in F_{k-1} \backslash F_{k}$, it follows that 
$u^{n} \notin F_{n(k-1)+1}$ for all $n \geq 1$. This shows that an equation of the form $u^{n}+a_{1} u^{n-1}+\cdots a_{n-1} u+a_{n}=0$, with $a_{i} \in F_{k}^{i}$, is impossible (because then $a_{n-i} u^{i} \in F_{(n-i) k} F_{i(k-1)} \subset F_{n k-i} \subset F_{n(k-1)+1}$ for all $\left.i=0, \ldots, n-1\right)$.

It is sufficient to show that $I^{* s p} \subset F_{k+1}$. Assume by contradiction that there is a $u \in I^{* s p}, u \notin F_{k+1}$. The assumption that gr $\mathcal{F}$ is reduced implies that $u^{q_{0}} \notin F_{k q_{0}+1}$ for every $q_{0}$.

Choose a fixed $c$ such that there exists a $q_{0}$ with $c u^{q_{0} q} \in \mathfrak{m}^{q} I^{\left[q_{0} q\right]}$ for all $q \gg 0$. Since the ideals $F_{1}^{[q]}$ are cofinal with $m^{q}$, one can re-adjust $q_{0}$ so that, taking into account the fact that $I^{\left[q_{0} q\right]} \subset\left(F_{k q_{0}}\right)^{[q]}$, we have

$$
c u^{q_{0} q} \in\left(F_{1+k q_{0}}\right)^{[q]} .
$$

Since $F_{k q_{0}+1}$ is integrally closed, we can choose $v$ a valuation such that $v\left(u^{q_{0}}\right)<v\left(F_{k q_{0}}+1\right)$. Apply $v$ to Equation 4 :

$$
v(c)+q v\left(u^{q_{0}}\right) \geq q v\left(F_{1+k q_{0}}\right) .
$$

Dividing by $q$ and taking limits, we get $v\left(u^{q_{0}}\right) \geq v\left(F_{1+k q_{0}}\right)$, which is a contradiction.

Note that Theorem 2.2 in $[\mathrm{Sm}]$ can be recovered as a particular case of Proposition 3.1: Smith's result assumes that $R$ is a graded normal finitely generated ring over a perfect field, and concludes that every homogeneous element $x$ in the tight closure of a homogeneous ideal $I$ generated by forms of degree at least $\delta$, but not in the ideal itself, must have degree at least $\delta+1$.

Corollary 3.2. Let $(R, \mathfrak{m})$ be an excellent Cohen-Macaulay normal local domain with minimal multiplicity, i.e. $e(R)=\operatorname{edim}(R)-\operatorname{dim}(R)+1$, and with perfect infinite residue field. If $\mathrm{g} r_{\mathfrak{m}}(R)$ is reduced, then $R$ is $F$ rational, i.e. all parameter ideals are tightly closed.

Proof. According to Theorem 4.2 (d) in $[\mathrm{HH}]$, it is enough to show that one parameter ideal is tightly closed. Let $\mathfrak{a}$ be a parameter ideal which is a minimal reduction of $\mathfrak{m}$. The minimal multiplicity assumption implies that $\mathfrak{m}^{2}=\mathfrak{m a}$, since

$$
e(R)=l\left(\frac{R}{\mathfrak{a}}\right)=l\left(\frac{R}{\mathfrak{m} \mathfrak{a}}\right)-l\left(\frac{\mathfrak{a}}{\mathfrak{m} \mathfrak{a}}\right) \geq 1+n-d,
$$

where $d=\operatorname{dim}(R), n=\operatorname{edim}(R)=\mu(\mathfrak{m})$, with equality if and only if $\mathfrak{m}^{2}=\mathfrak{m a}$. Thus we have $\mathfrak{m}^{2} \subset \mathfrak{a} \subset \mathfrak{m}$; according to Theorem 3.1, this implies that $\mathfrak{a}$ is tightly closed. 
Continuing along the same lines yields the following Proposition, which may be useful for the study of so-called big ideals (an ideal is big if every ideal containing it is tightly closed).

Proposition 3.3. Let $R, \mathcal{F}$ be as in Theorem 3.1; assume in addition that gr $\mathcal{F}$ is a domain. Let $I$ be an ideal of $R$ such that $F_{n+2} \subseteq I \subseteq F_{n}$ for some $n$. Let $f_{1}, \ldots, f_{t}, l_{1}+g_{1}, \ldots, l_{s}+g_{s}$ be a set of generators for $I$, with $f_{1}, \ldots, f_{t}, g_{1}, \ldots, g_{s} \in F_{n+1}$, and $l_{1}, \ldots, l_{s} \in F_{n} \backslash F_{n+1}$. Let $I_{0}$ be the ideal of gr $\mathcal{F}$ generated by the classes of $l_{0}, \ldots, l_{s}$ in $F_{n} / F_{n+1}$.

If $I_{0}$ is tightly closed in gr $\mathcal{F}$, then $I$ is also tightly closed.

Proof. It is enough to show that $I^{* s p} \subset I$. Let $u \in I^{* s p}$. By Theorem 3.1, we may assume that $u \in F_{n+1} \backslash F_{n+2}$, and as in the proof of Theorem 3.1, we can choose $c \in F_{\alpha} \backslash F_{\alpha+1}$ and $q_{0}$ such that $c u^{q} \in F_{q / q_{0}} I^{[q]}$ for all $q \geq q_{0}$.

In gr $\mathcal{F}$, this yields an equation of the form

$$
\overline{c u^{q}}=\beta_{1}{\overline{l_{1}}}^{q}+\cdots+\beta_{s}{\overline{l_{s}}}^{q}
$$

where the overline represents taking the class in $F_{\alpha+q(n+1)} / F_{\alpha+q(n+1)+1}$.

Since $I_{0}$ is tightly closed, this implies that $\bar{u} \in I_{0}$, where $\bar{u}$ represents the class of $u$ in $F_{n+1} / F_{n+2}$. Thus, $u \equiv a_{1} l_{0}+\cdots+a_{s} l_{s} \bmod F_{n+2}$, where $a_{1}, \ldots, a_{s} \in F_{1}$. Since $a_{i} l_{i} \equiv a_{i}\left(l_{i}+g_{i}\right) \bmod F_{n+2}$, it follows that $u \in I$.

Note that this proposition allows us to reduce showing that the nonhomogeneous ideal $I$ is tightly closed to showing that a homogeneous ideal $I_{0}$ is tightly closed.

Theorem 3.1 and Proposition 3.3 can be applied in particular for the case $F_{k}=\mathfrak{m}^{k}$, with the corresponding assumptions on the graded ring $\operatorname{gr}_{\mathfrak{m}} R$.

If in Proposition 3.3 one assumes instead that $R$ is graded normal domain, with $\mathfrak{m}$ denoting the maximal homogeneous ideal, and the filtration is $F_{k}=\mathfrak{m}^{k}$, the same proof shows that $I^{*} \subset I+I_{0}^{* s p}$ (remove the assumption that $I_{0}$ is tightly closed). 


\section{REFERENCES}

[Ab] I. Aberbach, Extensions of weakly and strongly F-rational rings by flat maps, J. Algebra, 241 (2001), 799-807.

$[\mathrm{H}] \quad$ N. Hara, A characterization of graded rational singularities in terms of of Frobenius maps, Amer. J. Math., 120 (1998), 981-996.

[HH] M. Hochster and C. Huneke, F-regularity, test elements, and smooth base change, Trans. Amer. Math. Soc., 346 (1994), 1-62.

[HS] C. Huneke and K.E. Smith, Kodaira vanishing and tight closure, J. reine angew. Math., 484 (1997), 127-152.

[MS] V. B. Mehta and V. Srinivas, A characterization of rational singularities, Asian J. Math., 1 (1997), 249-271.

[Sm] K. E. Smith, Tight closure in graded rings, J. Math. Kyoto Univ., 37 (1997), 35-53.

[Vr] A. Vraciu, *-Independence and special tight closure, J. Algebra, 249 (2002), $544-565$.

Craig Huneke

Department of Mathematics

University of Kansas

Lawrence, KS 66045

U.S.A.

huneke@math . ukans . edu

Adela Vraciu

Department of Mathematics

University of Kansas

Lawrence, KS 66045

U.S.A.

avraciu@math .ukans . edu 